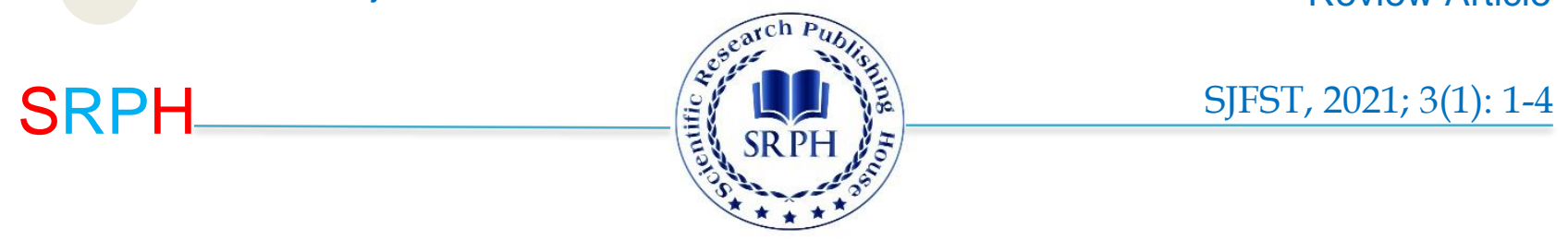

\title{
Dietary Risk Reduction Projects in Industrial Foods in Iran
}

\author{
Maryam Amini ${ }^{1 *}$, Azam Doustmohammadian ${ }^{2}$ and Mansour Ranjbar ${ }^{3}$
}

${ }^{1}$ Department of Nutrition Research, Faculty of Nutrition and Food Technology, National Nutrition and Food Technology Research Institute, Shahid Beheshti University of Medical Sciences, Tehran, Iran.

${ }^{2}$ Gastrointestinal and Liver Diseases Research Center, Iran University of Medical Sciences, Tehran, Iran.

${ }^{3}$ National Professional Officer, WHO.

\section{*Corresponding Author: \\ $邓$ Aminim@sbums.ac.ir}

Received: 25 November, 2020

Accepted: 15 January, 2021

Published: 30 January, 2021

\begin{abstract}
Non-communicable diseases (NCD) are the major cause of death worldwide. High intake of salt, sugar and fat are known as main risk factors for NCD. To address the issue some projects have been implemented in the country. This study aimed to seek for the initiatives targeting salt, sugar, and fat reduction in industrial foods and the status of implementation of food labeling. Meanwhile, we searched for studies which evaluated the above mentioned projects. Methods: We conducted an extensive review in domestic sources namely reports of national plans, protocols, dissertations, and progress reports of ongoing and terminated projects as well as rules and standards of Iran Food and Drug Administration (IFDA) and Institute of Standards and Industrial Research of Iran (ISIRI). Findings: There were several main projects in the country regarding reduction of dietary risk factors in industrial foods. Traffic light food labeling scheme and restrictions on imports of palm oil or food containing it, with support from food producers and revision of food standards were main interventions which were implemented by IFDA and ISIRI, respectively. In another project implemented by National Nutrition \& Food Technology Research Institute (NNFTRI) the previously mentioned projects were evaluated. Results of other related projects are presented, as well. Conclusion: Some important steps have taken to reduce the rate of NCDs in the country. However, to achieve the goal of $25 \%$ reduction in NCDs by 2025 , having a Surveillance, Monitoring and Evaluation (SME) plan is highly suggested to supports policymakers to take decision timely and correctly towards the enhancement of the effectiveness of the program.
\end{abstract}

Keywords: NCD, Dietary risk reduction, Initiative, Surveillance, Monitoring, Evaluation

\section{Introduction}

Non-communicable diseases (NCD) are the major cause of death worldwide. High intake of salt, sugar and fat are known as main risk factors for NCD. To the best of our knowledge mean daily intakes of salt, sugar and fat are more than the recommended level among Iranians and NCDs have been responsible to more than 38 million deaths and 16 million premature death in the world annual [1]. Alarming prevalence of NCDs across the world has made them of particular importance by Global Action Plan on NCDs and Sustainable Development Goals [2-4]. The High-Level Meeting on NCDs in the United Nations emphasized on the need of all government policy response to this dramatic problem, as well [5]. This staggering toll of
NCDs and premature mortality leads policy makers to pay attention and instigate action across the globe [6]. It is documented that NCDs rank among the first causes of mortality in Iran [7-10]. It is stated that $82.2 \%$ of NCDs' deaths in the country are due to cardiovascular diseases, cancers, chronic pulmonary diseases, and diabetes which in turn highlight the role of diet as a key risk factor [11].

The World Health Organization (WHO) priority areas for reducing non-communicable diseases include tobacco, alcohol, physical inactivity and unhealthy diet. Unhealthy diet generates a larger burden of disease than the other three risk factors combined. It accounts for an estimated 11.3 million deaths annually, compared with 2.1 million for low physical activity, 6.1 million for tobacco smoke and 3.1 million for alcohol and drug use (12). The problem predominantly reflects an unhealthy global food environment dominated by 
processed foods high in sugar, salt, saturated fats and, industrial Trans Fatty Acids (TFAs).

In our country, the prevalence of nutrition related NCDs, due to direct and indirect consumption of sugar, salt and total fat is increasing. It is documented that consumption of sugar and fat in Iran are about 23 $\mathrm{kg} /$ per capita/year and $17 \mathrm{~kg} / \mathrm{per}$ capita/year, respectively which is $4.5 \mathrm{~kg}$ more that the global average. In addition, the level of salt intake in $41.2 \%$ of the population is at least twice as much is recommended by the WHO and the mean intake of salt in the country is $9.52 \mathrm{~g} /$ day for adults $[13,14]$.

Iran is among few countries with national action plan for prevention and control of NCDs, with specific target of reducing $25 \%$ by 2025 . To achieve the goal some projects have been implemented in the country. This study aimed to seek status of implementation of the initiatives targeting salt, sugar, and fat reduction in industrial foods and the status of implementation of food labeling. Meanwhile, we searched for studies which evaluated the above mentioned projects.

\section{Methods}

We conducted an extensive review in domestic sources namely reports of national plans, protocols, dissertations, and progress reports of ongoing and terminated projects as well as rules and standards of Iran Food and Drug Administration (IFDA) and Institute of Standards and Industrial Research of Iran (ISIRI).

\section{Findings}

There were several main projects in the country regarding reduction of dietary risk factors in industrial foods. Traffic light food labeling scheme and restrictions on imports of palm oil or food containing it, with support from food producers and revision of food standards were main interventions which were implemented by IFDA and ISIRI, respectively. In another project implemented by National Nutrition \& Food Technology Research Institute (NNFTRI) the previously mentioned projects were evaluated. Results of other related projects are presented, as well.

\section{National initiatives for reduction of dietary risk factors in industrial foods}

There are some national plans for initiatives aimed at reducing the nutrition risk factors of noncommunicable diseases in industrial foods in the country.

The first plan entitled "labeling of food and beverages" is being carried out by IFDA and MOHME since 2010. Based on this plan, all producer and importer of packaged foods are required to include nutritional labels with colorful markers resembling traffic light.
Required information per serving (or per 100 grams $/ 100 \mathrm{ml}$ of food products for which specific servings are not defined) includes energy; salt, total fat, trans fatty acids and sugar(s) (mono, di, other than polyols). Manufacturers are required to insert descriptive words "low", "moderate" and "high" in the markers .According to the criteria green, orange and red indicate low, medium and high risk factor content, respectively. Iran is the first country in the Eastern Mediterranean region which has accomplished to implement nutrition traffic light labelling and now implementation of traffic light nutritional labeling has $100 \%$ coverage [15].

The second plan has been carried out by ISIRI and was aimed to reduce nutritional risk factors through revision of national food standards. In the plan, 27 health-related standards have been revisedsince 2015 . The revised food products include vegetable butter, minarine, household oil, frying oil, confectionary and flour oil, nectar, juice, syrup, soft drinks, noncarbonated drinks, cake powder, roasted bread, soup noodle, rice noodle, processed seeds , canned olive, ketchup and tomato paste. In the revised standards, the content of edible oils and fats, sugar and salt in the food products have been modified [16].

\section{Other studies}

National plan of monitoring nutritional risk factors for non-communicable diseases in industrial and commercial foods was a project implemented by NNFTRI. In the project sugar, salt, total fat, saturated and trans fatty acids in industrial and commercial foods were being monitored. The under study food subgroups included drinks and non-meat canned foods; milk and dairy products; breads and cereal products; oil and fat; and meat and processed meats. In this study compliance of dietary risk factors content with labels of food packs in highly consumed industrial products was analyzed. In other words, the effectiveness of the two projects was evaluated by this study . According to the results, among the ready to eat drinks soft drinks/synthetic juices, and nectars), the lowest and highest total sugar content was for non-alcoholic malt beverages and nectars, respectively. The amount of sucrose in $82.11 \%$ of industrial dairy products did not comply with the national standard and the total sugar content of any of the products did not match the packaging claims. About $86 \%$ of food products had traffic light labels, among which salt content of $24.42 \%$ were and salt content of $61.63 \%$ were not in compliance with the labels. About $14 \%$ of the products did not have any traffic light label (Figure 1) [17].

Some researchers evaluated the consumer's awareness and literacy on food labels and traffic lights. The first cross-sectional study was undertaken in 2016 on 803 and 667 students to evaluate food label literacy in elementary and high schools in Iran. In addition, to 
determine to what extent food label literacy had been addressed in the text-books of primary and high school students their content were analyzed. Data were taken from a large study of Food and Nutrition Literacy (FNLIT) funded by NNFTRI. Results showed that a high proportion of primary and high school students had low level of food label literacy $(76.0 \%$ and $45.4 \%$, respectively) which means ability to obtain, process, and understand nutrition information on food labels to make appropriate diet-related decisions. The content analyses of elementary and high school textbooks showed that only one textbook (Sciences book of Sixth graders) had introduced food labels and only in regard to energy calculation. Low food label literacy was more frequent in elementary students compared to adolescents. It did not necessarily indicate the efficacy of educational system rather was due to more exposure and experience of the adolescents as they grow up [1820].

In a qualitative study to explore the consumers' perception on nutritional traffic light, after more than two years of implementation of the plan, the results indicated that $67.5 \%$ of the participants did not know the nutritional traffic light on food products. The most important ways that participants acquired their information about the nutritional traffic light were television, newspapers, cyber world, parents, advertisement and the Internet. The most frequent perceptions of people who were aware of the traffic light included:

- The traffic light indicates badness or goodness of the nutrients; colors of the labels imply important items; indicates basic information of foods; red color means harmfulness, bad for health; help to have better choice; green color means little harm.

- Some of the participants were satisfied by traffic light components, some believed shape of nutrition facts tables should be modified and some thought it was standard, healthy and beautiful.

- The reasons expressed by the participants for not paying attention to the traffic light included: lack of knowledge about the colors' concepts, having no substitution for food products which had red color in their labels, not having enough time to act based on the traffic light messages, small size of labels on packages, and not being familiar with food labeling because of negligence of mass media to introduce them.

The participants referred to some drawbacks which caused them not to focus on traffic light labels properly. They included disproportion of the traffic light colors with the background color, graphical problem, complexity of color with written information, illegibility, not attracting due to poor print quality, and misunderstanding of colors' concepts [21].

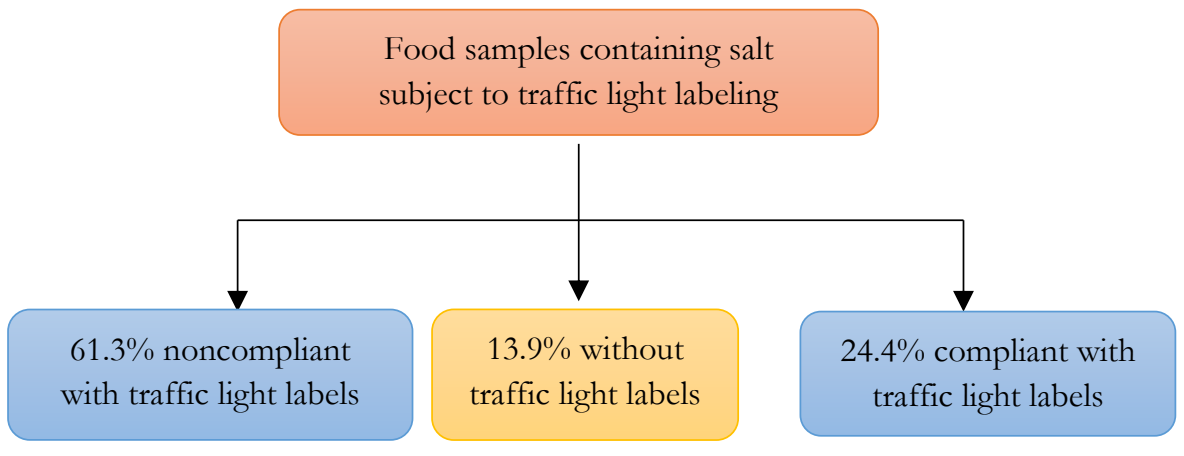

Figure 1. Compliance with traffic light labels.

\section{Discussion}

According to the review there were several national plan including "traffic light" food labeling scheme; salt, sugar, fat regulation, and restrictions on imports of palm oil or food containing it, with support from food producers, and revision of several national standards, since 2016 which aimed to decline dietary risk factors and subsequently NCDs in a national level.

As mentioned earlier, Iran is to reduce NCDs $25 \%$ in less than 5 years. Therefore, there is a need to define the national requirements and to develop the national plan for surveillance, monitoring and evaluation in the relevant area. National monitoring and evaluation experts need to be part of any multi-sectoral team working on plan implementation, and should take the lead in designing and carrying out evaluation activities.

Having a Surveillance, Monitoring and Evaluation (SME) plan is highly suggested to supports policymakers to take decision timely and correctly towards the enhancement of the effectiveness of the program. It is necessary to measure important indicators and to address critical topics including the coverage of interventions, alignment of the foods with the label and traffic light scheme, output and outcome of the interventions and trend in a period of time, impact of interventions on consumers, market, producers/importer approach and sustainability of interventions. 


\section{References}

1. Geneva, World Health Organization. Global status report on NCDs. 2014; www.who.int/nmh/publications/ncd-status-report2014/en/

2. Geneva, World Health Organization. Global action plan for the prevention and control of noncommunicable diseases 2013-2020. 2013; who.int/nmh/events/ncd_action_plan/en/

3. Sustainable Development Goals. New York, The United

Nations.

http://www.sustainabledevelopment.un.org/?Menu

$=1300$

4. Alwan A. The world health assembly responds to the global challenge of non-communicable diseases. E Mediterr Health J. 2013; 19(6): 511.

5. UN General Assembly. Political declaration of the high-level meeting of the general assembly on the prevention and control of non-communicable diseases. A/66/L.1. New York, The United Nations. 2011; http://www.un.org/ga/search/view_doc.asp?symbol= A/66/L.1

6. Hunter DJ, Reddy KS. Noncommunicable diseases. New Eng J Med. 2013; 369(14): 1336-1343.

7. Haagsma JA, Graetz N, Bolliger I, Naghavi M, Higashi H, Mullany EC. The global burden of injury: incidence, mortality, disability-adjusted life years and time trends from the Global Burden of Disease study 2013. Inj Prev. 2016; 22(1): 3-18.

8. Geneva, World Health Organization. NonCommunicable Diseases (NCD) Country Profiles. 2014; $\quad$ http://who.int/nmh/countries/irn_en. pdf?ua $=1$

9. Collaboration NRF. Trends in adult body-mass index in 200 countries from 1975 to 2014: A pooled analysis of 1698 population-based measurement studies with 19. 2 million participants. Lancet. 2016; 387(10026): 1377-1396.

10. Collaboration NRF. Worldwide trends in diabetes since 1980: A pooled analysis of 751 population-based studies with 4. 4 million participants. Lancet. 2016; 387(10027): 1513-1530.

11. Global Burden of Diseases. Washington, Institute of Health Metric and Evaluation. 2014; http://vizhub.healthdata.org/gbd-compare/
12. Gakidou E, Afshin A, Abajobir AA, Abate KH, Abbafati C, Abbas KM. Global, regional, and national comparative risk assessment of 84 behavioural, environmental and occupational, and metabolic risks or clusters of risks, 1990-2016: A systematic analysis for the Global Burden of Disease Study 2016. Lancet. 2017; 390(10100): 1345-1422.

13. Organization WH. Global action plan for the prevention and control of noncommunicable diseases 2013-2020. 2013.

14. Rezaei S, Mahmoudi Z, Sheidaei A, Aryan Z, Mahmoudi N, Gohari K. Salt intake among Iranian population: the first national report on salt intake in Iran. J Hypertens. 2018; 36(12): 2380-2389.

15. Zargaraan A, Dinarvand R, Hosseini H. Nutritional traffic light labeling and taxation on unhealthy food products in Iran: health policies to prevent noncommunicable diseases. Iran Red Crescent Med J. 2017; 19(8).

16. Institute of Standards and Industrial Research of Iran (ISIRI). http:/ / standard.isiri.gov.ir/

17. National plan of monitoring nutritional risk factors for non-communicable diseases in industrial and commercial foods. Final report, National Nutrition and Food Technology Research Institute (NNFTRI). 1398.

18. Doustmohammadian A, Omidvar N, Ashoori M, Eini-Zinab H, Dehghani M. Food label literacy in children and adolescents in Iran: its correlates and outcomes. Nutr Food Sci Res. 2018; Special Issue(5): Supplement 1:66.

19. Doustmohammadian A, Keshavarz Mohammadi N, Omidvar N, Amini M, Abdollahi M, Eini-Zinab H. Food and nutrition literacy (FNLIT) and its predictors in primary school children in Iran. Health Promot Int. 2018.

20. Arvand A, Dehghani M, Omidvar N, Ashoori M. Food and nutrition literacy: A neglected aspect in high school textbooks in Iran. Iran J Nutr Sci Food Tech. 2018; 13(4): 29-38.

21. Haghighian Roudsari A, Zargaran A, Milani Bonab A, Abdollah SF. Consumers' perception of nutritional traffic light in food products: A qualitative study on new nutritional policy in Iran. Nutr Food Sci Res. 2018; Special Issue(5): Supplement 1:69.

\section{SJFST}

Copyright: (C) 2021 The Author(s); This is an open-access article distributed under the terms of the Creative Commons Attribution License (http://creativecommons.org/licenses/by/4.0), which permits unrestricted use, distribution, and reproduction in any medium, provided the original work is properly cited.

Citation: Amini M, Doustmohammadian A, Ranjbar M. Dietary Risk Reduction Projects in Industrial Foods in Iran. SJFST, 2021; 3(1): 1-4.

https://doi.org/10.47176/sjfst.3.1.1 